\title{
PAPER
}

\section{Managing severe lower limb spasticity in multiple sclerosis: does intrathecal phenol have a role?}

\author{
L Jarrett, P Nandi, A J Thompson
}

J Neurol Neurosurg Psychiatry 2002;73:705-709

See end of article for authors' affiliations

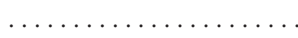

Correspondence to: Professor A J Thompson, Institute of Neurology, Queen Square, London WC1 N 3BG, UK;

a.thompson@ion.ucl.ac.uk

Received 24 April 2002 In revised form 17 July 2002

Accepted 15 August 2002

\begin{abstract}
Objective: Established treatment options for managing severe lower limb spasticity and associated pain are of limited value in people with advanced multiple sclerosis (MS). This has resulted in a resurgence of the use of lumbar intrathecal phenol injection (IP). The aim of this study was to investigate the authors' experience with IP.

Methods: This observational study collected cross sectional data from patients with progressive MS who received IP for severe lower limb spasticity. Data from 25 patients were collected prospectively before and after treatment. In 15 cases the data related to the first treatment and in 10 to serial injections. Outcome measures collected included the Ashworth scale, a spasm frequency scale, a pain rating score, and the percentage achievement of practical goals.

Results: After injection, all patients demonstrated reduced lower limb tone bilaterally. After the initial injection there was significant improvement on the targeted as compared with the non-targeted side (Wilcoxon rank $p=0.003$ ), while no difference in the degree of improvement between the targeted and non-targeted side was seen after serial injections (Wilcoxon rank $p=0.731$ ). Twenty four patients were easier to position and 21 had a reduction in their spasm frequency and intensity. Eleven patients with pain reported benefit. Carers found washing and dressing easier in 16 patients and improved safety when using the hoist in 10 . Six patients had recurrence of skin breakdown and five patients reported transient adverse changes in their bowel function.

Conclusions: IP can reduce lower limb tone bilaterally after both initial and serial injections. This is most noticeable on the targeted side after initial injection. IP can reduce spasms and pain, leading to improvements in care and overall comfort. IP is an effective treatment option in the management of severe spasticity. Documented selection criteria are essential.
\end{abstract}

S pasticity in multiple sclerosis (MS) is common, tends to worsen as disability progresses, and is often associated with bladder and bowel dysfunction. In a recent survey of people with MS, 74\% reported symptoms of spasticity and $78 \%$ felt their quality of life was moderately or severely affected as a direct result of this symptom. ${ }^{1}$

Intrathecal phenol (IP) was first reported to reduce pain and spasticity in the 1950 s. $^{2-4}$ However, complications such as bladder and bowel incontinence, limb weakness, and painful paraesthesia were highlighted, although it was not clear if these complications resolved or persisted. ${ }^{5}$ Furthermore, the effect of IP seemed to diminish over time with pain and spasms returning. ${ }^{-9}$ These issues, together with the introduction of intrathecal baclofen (ITB) in the late eighties and more recently botulinum toxin, have contributed to the relative neglect of IP as a management option. However, in appropriately selected people it may be a highly effective and well tolerated treatment in the management of severe spasticity and associated pain.

The primary effect of phenol on nervous tissue is coagulation and denaturing of proteins, which leads to cellular and axonal damage. ${ }^{10}$ Initially it was postulated that IP might act selectively on spasticity and pain without affecting the nerves responsible for bladder and bowel function. ${ }^{34}$ However, it became apparent that phenol damages axons non-selectively, irrespective of their size, thereby affecting motor and sensory nerve fibres indiscriminately. ${ }^{910}$ The magnitude of the effect depends on the volume and concentration of phenol used. Solutions of between $5 \%-8 \%$ phenol produce a relatively neuroselective effect whereas higher concentrations can cause extensive fibrosis and thickening of the arachnoid mater within the subarachnoid space. ${ }^{10}$ Administering phenol in glycerin renders it hyperbaric and viscous, resulting in limited spread, and hence localised nerve injury. ${ }^{6}$
This paper presents the results of cross sectional data from an observational study of IP in 25 patients with MS.

\section{METHODS}

Twenty five people with MS and severe lower limb spasticity were selected for IP treatment using defined criteria (table 1). Written and verbal information on the treatment and its effects were given, by both the medical and nursing team, consent was obtained by the pain management consultant anaesthetist. The group included patients having an initial injection (initial group) and those who were having subsequent injections (serial group). As part of routine practice a structured programme for assessing spasticity, incorporating goals and outcome measures including the Ashworth Scale, ${ }^{12}$ a spasm frequency scale, ${ }^{13}$ and a global pain rating score ${ }^{14}$ was used on day of admission and repeated within 24 hours after each treatment. This included an individualised goal agreed, between the team, the patient, and, if appropriate, their carers. After treatment, the same people evaluated whether the goal had been achieved. The Ashworth Scale was used to measure tone and ease of passive movement in the lower limbs. Five muscle groups on both the targeted and non-targeted side were measured: the hip and knee extensors, hip and knee flexors, and ankle dorsiflexors. Before and after injection the median and interquartile ranges of the Ashworth score for each of the defined muscle groups was calculated for all patients. A spasm frequency score was used before and after treatment. Carers were asked to report the number of spasms

Abbreviations: MS, multiple sclerosis; IP, intrathecal phenol 


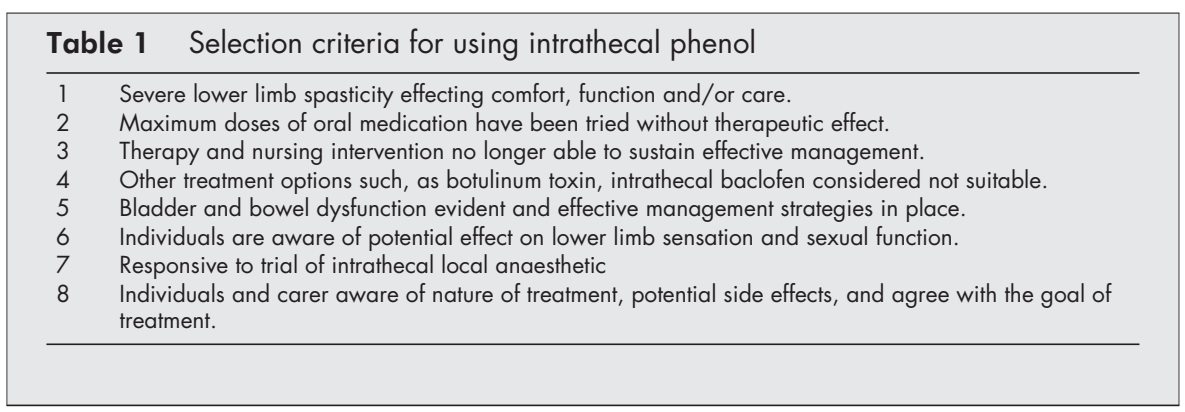

witnessed if patients were unable to give an account and the patient or carer subjectively commented on spasm intensity. Pain was evaluated before and after treatment using a pain rating scale. Particpants were asked to rate their pain over the preceding 24 hours for each leg between 0 (no pain) to 100 (maximum pain). ${ }^{14}$

\section{Procedure technique}

Before the phenol treatment, a local anaesthetic test injection of hyperbaric bupivacaine $0.5 \%$, between 1.5 and $2.5 \mathrm{ml}$ was injected via a 24 gauge Sprotte spinal needle with introducer. At least 24 hours elapsed between the test and treatment injection to give sufficient time for the local anaesthetic to wear off and the patient to confirm their wish to proceed. The definitive treatment injection-5\% phenol in glycerol-was administered via an 18 gauge standard (Quincke point) spinal needle. All the injections were carried out by the same operator $(\mathrm{PN})$ using an aseptic technique. Intravenous access was obtained and maintained throughout the procedure. Blood pressure, pulse, and respirations were monitored throughout the procedure and for two hours after treatment. The injections were carried out at level $\mathrm{L} 2 / 3$ or $\mathrm{L} 3 / 4$, aiming to reduce hip spasticity, flexor, adductor, and extensor spasms. The volume injected was between 1.5 and $2.5 \mathrm{ml}$. The patient was placed on the side to be targeted in a modified lateral position with $30^{\circ}-40^{\circ}$ of pronation and the lumbar spine horizontal as judged by external landmarks (fig 1). After the injection, this position was maintained for 20 minutes to promote relatively selective distribution of the injectate to the anterior roots. Relative selectivity of motor denervation is desirable to minimise deleterious effects on bladder, bowel, and sexual function.
Follow up injections were required if there was a limited effect on the targeted side or if the other side also required treatment. These were arranged on an outpatient basis at least six weeks later, to permit adequate time to assess the affect of the injection and concurrent interventions such as physiotherapy and improved seating.

\section{Statistical analysis}

The Wilcoxon signed ranks test was used to compare the Ashworth and spasm frequency scores before and after treatment.

\section{RESULTS}

Seventeen women and eight men received IP, 15 in the initial and 10 in the serial group. All patients had progressive MS. The age ranged from 32 to 65 years with a mean of 47.76 years and an Expanded Disability Status Scale ${ }^{15}$ of 8.0 or above, indicating that patients were either wheelchair or bed dependent. Outcome measures were recorded before and after treatment in both initial and serial groups. The mean number of injections in the serial group was 3.2 (range 2-9).

The individual goals, which related to improved comfort and practical management, such as hoisting, positioning, washing, and dressing were all achieved. Twenty four patients, including all in the initial group were easier to position in bed and their wheelchairs. Sixteen patients stated it was easier for their carers to carry out lower half washing and dressing. Carers reported that 10 people were safer to hoist and one person was able to reduce the number of his carers from three or two to one.

All patients demonstrated a significant reduction in their Ashworth scores in all the tested muscle groups for the targeted and non-targeted injection sides, after both initial

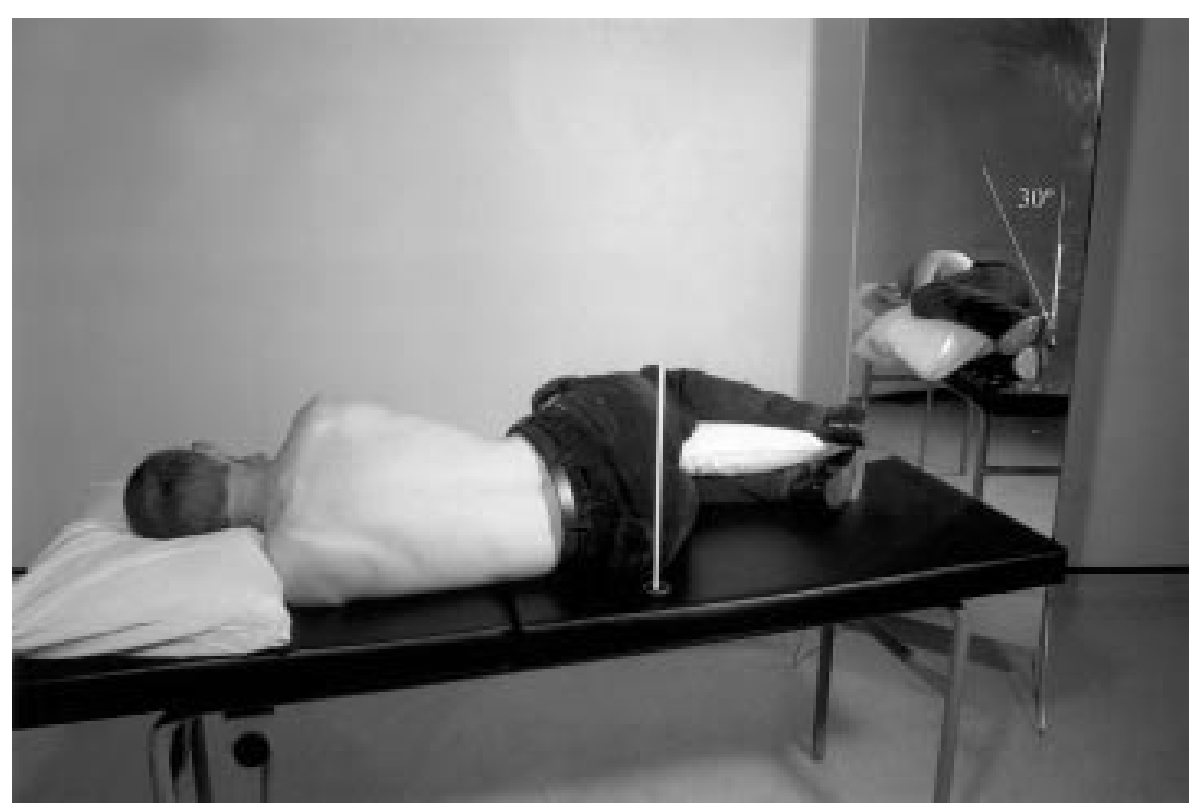

Figure 1 Modified lateral position with $30^{\circ}$ pronation. 


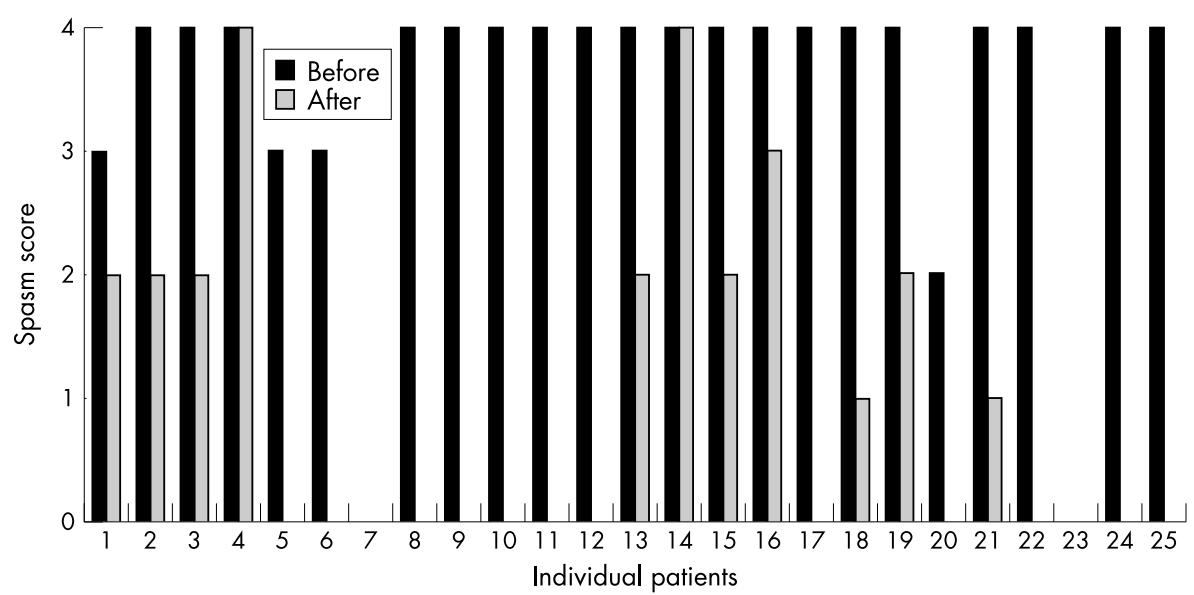

Figure 2 Frequency of spasms before and after intrathecal phenol.

Table 2 The median and interquartile ranges (IQR) for each muscle group and the significance between targeted and non-targeted sides (Wilcoxon signed ranks test $p<0.05$ )

\begin{tabular}{|c|c|c|c|c|c|c|c|c|c|}
\hline \multirow[b]{2}{*}{ Muscle group } & \multicolumn{4}{|l|}{ Targeted leg } & \multicolumn{4}{|c|}{ Non-targeted leg } & \multirow{2}{*}{$\begin{array}{l}\mathrm{p} \text { Value for } \\
\text { difference in } \\
\text { improvement } \\
\text { between legs }\end{array}$} \\
\hline & $\begin{array}{l}\text { Before median } \\
\text { (IQR) }\end{array}$ & $\begin{array}{l}\text { After median } \\
\text { (IQR) }\end{array}$ & $\begin{array}{l}\text { Median } \\
\text { change }\end{array}$ & $\begin{array}{l}\text { p Value } \\
\text { before/aft er } \\
\text { change }\end{array}$ & $\begin{array}{l}\text { Before median } \\
\text { (IQR) }\end{array}$ & $\begin{array}{l}\text { After median } \\
\text { (IQR) }\end{array}$ & $\begin{array}{l}\text { Median } \\
\text { change }\end{array}$ & $\begin{array}{l}\text { p Value } \\
\text { before/aft } \\
\text { er change }\end{array}$ & \\
\hline \multicolumn{10}{|c|}{ Initial treatment group } \\
\hline Flexor & $3(2-3)$ & $0(0-1)$ & 2 & 0.001 & $3(2-4)$ & $2(1-3)$ & 1 & 0.076 & 0.02 \\
\hline Extensor & $3(2-3)$ & $0(0-1)$ & 2 & 0.001 & $3(2-3)$ & $2(1-3)$ & 0 & 0.016 & 0.01 \\
\hline Dorsiflexor & $3(1-3)$ & $0(0-2)$ & 1 & 0.003 & $3(2-3)$ & $2(0-3)$ & 2 & 0.01 & 0.02 \\
\hline \multicolumn{10}{|c|}{ Serial treatment group } \\
\hline Flexor & $2(2-3.25)$ & $0.5(0-1)$ & 2 & 0.006 & $2.5(1.75-3)$ & $0(0-1)$ & 2 & 0.007 & 0.78 \\
\hline Extensor & $2.5(1.75-3)$ & $1(0-1.25)$ & 1.5 & 0.007 & $2.5(1-3)$ & $0.5(0-1)$ & 1.5 & 0.007 & 0.52 \\
\hline Dorsiflexor & $2.5(0.75-3)$ & $0(0-1)$ & 1.5 & 0.016 & $2(0.75-3)$ & 0.5 (0-1.25) & 1 & 0.056 & 0.50 \\
\hline
\end{tabular}

and serial injections (Wilcoxon rank $\mathrm{p}<0.05$, table 2 ). The reduction in tone was greater on the targeted side when compared with the non-targeted side after the initial treatment (Wilcoxon rank $\mathrm{p}<0.05$, table 2 ), whereas the reduction was similar on the two sides in the serial treatment group (Wilcoxon rank $\mathrm{p} \geqslant 0.5$, table 2 ). Serial injections produced the lowest median Ashworth scores. All 23 patients with spasms reported a reduction in intensity, and 21 reported a reduction in frequency (fig 2). The median spasm score of the group before treatment was 4 (IQR 4-4) and after treatment was 0 (IQR $0-2$ ). This was statistically significant (Wilcoxon $\mathrm{p}$ $=$ or $<0.001)$. Patients presented with a mixed pattern of flexor, extensor and/or adductor spasms, and when spasms persisted, the pattern was unchanged.
Eleven patients had pain before treatment ( 10 had no pain before or after injection and four were cognitively impaired to a degree that made them unable to use the analogue scale). Of the 11, after treatment, four had no pain in either leg. Seven had reduced pain in the target leg, for three it was totally ablated (fig 3 ) and six had reduced pain on the non-target side.

Ten patients had a previous history of skin breakdown and after injection six had subsequent broken skin areas. Five patients reported transient adverse changes in their bowel function (three after subsequent treatments, two after one treatment). Two reported faecal incontinence, which lasted between two to four weeks but then resolved, three reported constipation such that two needed to increase the strength of the enemas they used regularly, and one required manual

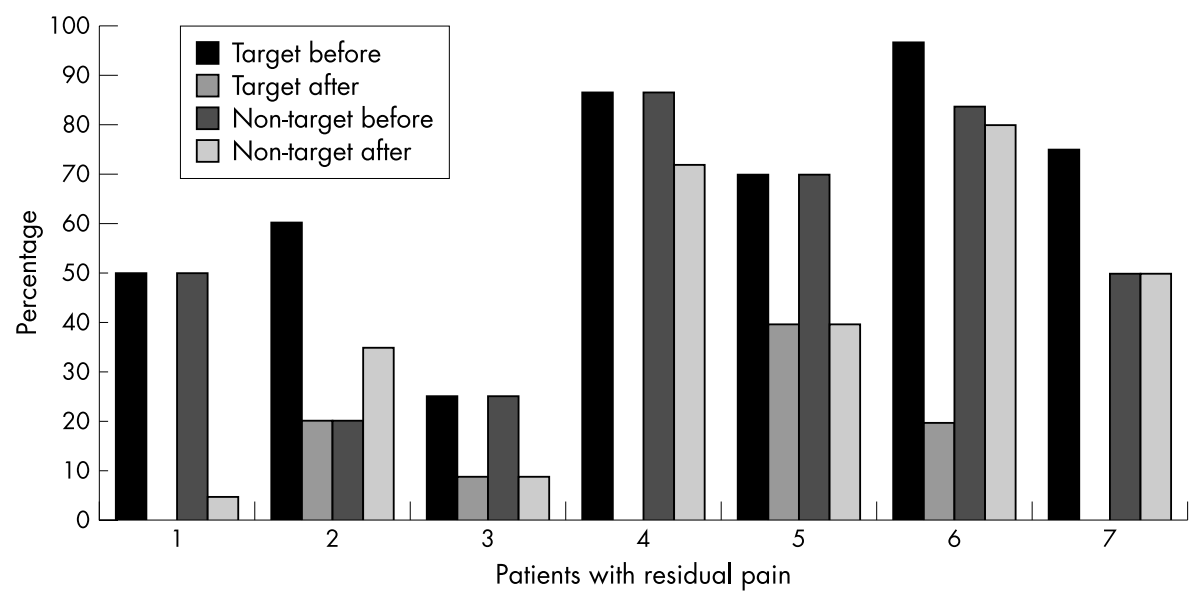

Figure 3 Before and after targeted and non-targeted pain levels in participants with pain. 


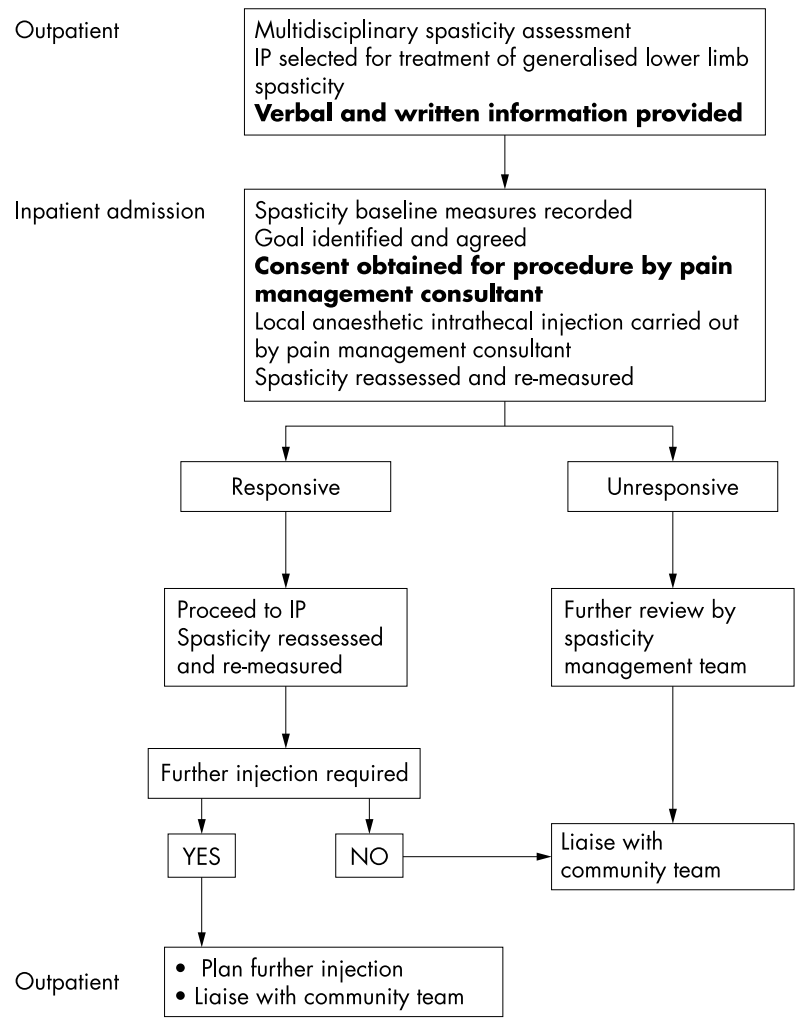

Figure 4 Intrathecal phenol treatment algorithm for managing lower limb spasticity.

evacuations for four weeks before returning to their previous management strategy.

None of the group reported adverse effects on their bladder or sexual function. Two men concerned about their ability to have erections chose to have a lower dose $(1.5 \mathrm{ml})$, in an effort to minimise the risk. Two women reported enhanced sexual function because of improved lower limb flexibility.

A moderate drop in blood pressure after the phenol injection was seen in some cases however this did not require active management, other than slowly raising them into sitting over a period of one to two hours. There was no case of adverse outcome. Twenty four patients have required more than one injection, 13 to achieve a bilateral effect, 11 because of a wearing off of effect.

\section{DISCUSSION}

Reduction in tone in the flexor, extensor, and dorsiflexor muscles of both lower limbs was seen after each injection of IP. In the initial treatment group there was greater reduction in tone on the targeted side, and no significant difference between the targeted and non-targeted sides was seen after serial injections. Treatment in the serial group resulted in significantly lower levels of tone on the non-targeted side, suggesting that benefit may be extended with serial injections resulting in a further reduction in of tone. IP reduces the intensity of spasms and pain, although type of spasm and frequency may not be affected. Perhaps most importantly, IP improved care relating to positioning, hoisting, washing, and dressing.

\section{Procedure}

A preliminary trial of intrathecal local anaesthetic was instructive in the decision making process for three reasons:

(1) It gave the patient the opportunity to experience the probable effect of the definitive procedure, while having only a transient effect. This experience was key to enabling patients to decide whether they wished to proceed with the IP.
(2) It permitted shortened soft tissue or contractures to be identified that might, if the person was injected, restrict positioning or ease of care. This enabled the team to plan specific treatments after injection relating to physiotherapy, nursing, and wheelchair seating requirements to promote goal achievement.

(3) It gave some indication of the probable spread and effect of phenol. When given with glycerol, phenol is viscous and will not spread as much as the local anaesthetic. Therefore the effect of the local anaesthetic is more extensive; hence if no undesired effects are seen then they are considered unlikely to occur with IP.

The volumes of phenol used were larger than those quoted in earlier studies. ${ }^{23}$ In the authors' experience, results are usually suboptimal with volumes less than $1.5 \mathrm{ml}$. Serial injections may be required to achieve a bilateral effect or if spasticity or spasms return. This wearing off of effect could be explained by the possibility that anterior horn cells are not damaged by phenol administered in this way ${ }^{16}$ and that damage is restricted to the motor axons.

\section{Adverse effects}

Five patients experienced changes in their bowel function. This emphasises the need to screen people before injection to ensure they have effective management strategies in place, which can subsequently be adapted, if transient problems occur after treatment. Four of the patients had subsequent injections as they stated the advantages of reduced spasticity outweighed the transient effect on their bowel management.

As IP damages sensory nerves, it might theoretically be a risk factor for skin breakdown and delayed healing of existing, broken skin. It is difficult to conclude from the data in this study whether IP adversely affects skin integrity. There was no evidence to suggest that those with intact skin before injection were compromised after injection. However, it may be good practice to only use IP if the person meets the selection criteria (table 1), has an effective skin management regimen, and their carers are aware of the potential risks.

\section{Outcomes}

The use of goals agreed between the patient, carers, and team permitted the patients' or carers' perspective to be the focus of treatment. All goals were directed towards improving positioning, comfort, or ease of carrying out an individual's daily care needs. The outcome measures used provided evidence of effectiveness, but as spasticity is complex the addition of further measures may provide a more comprehensive picture. For instance, although the majority of the group had no pain they expressed an improvement in overall comfort, because of improved ease of care and positioning. The addition of an overall comfort rating score similar to the pain score has been introduced to assess this: 0 (very uncomfortable) to 10 (very comfortable). It was found that asking the question rather than using visual analogues was easier for this highly disabled group as they had difficulty seeing the scale or did not have the manual dexterity to make a mark on the line. To strengthen the data collected, the addition of disability (Bartel Index ${ }^{17}$ ) or carer burden measures (Carer Strain Index ${ }^{18}$ ) could be considered.

\section{Intrathecal phenol or intrathecal baclofen?}

It is important to clarify the role of IP alongside other treatments, such as oral or other intrathecal drugs, inpatient or outpatient therapy, or multidisciplinary rehabilitation. This applies particularly to ITB, which is also used in the management of severe generalised lower limb spasticity. ${ }^{13}{ }^{19}$ ITB can permit greater flexibility over the short-term and longer term. This is important to achieve a balance between maintaining functional abilities and treating the fluctuating pattern of spasticity, seen in patients with a progressive disease such as MS. IP may be preferable to ITB if the patient, does not 
adequately respond to ITB at the trial stage, ${ }^{19}$ over time, ${ }^{2021}$ develops tolerance, ${ }^{22}$ or does not want the responsibilities associated with managing a pump implant. These include regular pump refills, taking responsibility for seeking help if alarms sound, or side effects occur. IP may however be more appropriate at a later stage of disease than ITB. Although IP requires an expert injection, the individual responsibility is less and no other costly or special equipment is required.

\section{CONCLUSIONS}

These findings show that IP was an effective treatment in the management of lower limb spasticity in 25 people with MS when used as part of an overall programme, which included expert multidisciplinary assessment, selection, ongoing treatment, education, measurement, and injection. Multidisciplinary assessment was key to effective patient selection and has led to the development of a defined treatment algorithm (fig 4).

When considering long term management the infrastructure for repeat assessments, injections, ongoing monitoring of skin integrity, bladder and bowel management strategies need to be considered. Future studies on the effect of serial IP: on long term management of lower limb spasticity, skin integrity, sexual function, and on the quality of life of patients with MS and their carers would be beneficial.

\section{ACKNOWLEDGEMENTS}

We thank physiotherapists D Richardson, L Lockley, J Neale, clinical nurse specialist pain management $\mathrm{D}$ Colbeck and clinical nurse specialist multiple sclerosis Bernadette Porter (National Hospital for Neurology and Neurosurgery, UCLH Hospital Trust, Queen Square, London) for assistance in assessment and data collection.

\section{Authors' affiliations}

L Jarrett, P Nandi, A J Thompson, National Hospital for Neurology and Neurosurgery, University College London Hospitals, London, UK A J Thompson, Institute of Neurology, University College London, London, UK

Competing interests: none declared.

\section{REFERENCES}

1 MS Society. MS Symptom Management Survey. London: MS Society, 1999.

2 Maher RM. Relief of pain in incurable cancer. Lancet 1955;i:18-20.

3 Kelly RE, Gautier-Smith PC. Intrathecal phenol in the treatment of reflex spasms and spasticity. Lancet 1959; ii: 1 102-5.

4 Nathan PW. Intrathecal phenol to relieve spasticity in paraplegia. Lancet 1959;ii:1099-102.

5 Nathan PW, Scott TG. Intrathecal phenol for intractable pain. Lancet 1958;i:76-80.

6 Wood KM. The use of phenol as a neurolytic agent: A review. Pain 1978:5:205-29.

7 Lourie H, Vanasupa P. Comments on the use of intraspinal phenolpantopaque for relief of pain and spasticity. J Neurosurg 1963;20:60-3.

8 Hansebout R, Cosgrove JBR. Effects of intrathecal phenol in man. A histological study. Neurology 1966;16:277 -82.

9 Nathan PW. Treatment of spasticity with perineural injections of phenol. Dev Med Child Neurol 1969;11:384

10 Bonica JJ. Neurolytic blockade and hypophysectomy. In: Bonica JJ, ed. The management of pain. 2nd edn. London: Lea and Febiger, 1990:1980-83.

11 Harris $\mathbf{P}$, Simpson JA. A new technique for the use of phenol to control spasticity and spasms. Paraplegia 1963:1:197-8.

12 Ashworth B. Preliminary trial of carisoprodol in multiple sclerosis. Practitioner 1964:192:540-2.

13 Penn RD, Savoy SM, Corcos D, et al. Intrathecal baclofen for severe spinal spasticity N Engl J Med 1989;320:1517-21.

14 Pierson SH. Outcome measures in spasticity management. Muscle Nerve 1997; (suppl 6):S36-60.

15 Kurtzke JF. Expanded Disability Status Scale. Neurology 1983;33:1444-52.

16 Gelber DA, Jozefczyk PB. The management of spasticity in multiple sclerosis. International Journal of MS Care 1999;1:1-15.

17 Mahoney FI, Barthel DW. Functional evaluation: the Barthel index. Md State Med J 1965;14:61-5.

18 Robinson BC Validation of a caregiver strain index. J Gerontol 1983;38:344-8.

19 Jarrett L, Leary SM, Porter B, et al. Managing spasticity in people with multiple sclerosis. A goal orientated approach to intrathecal baclofen therapy. International Journal of MS Care 2001;3:2-10.

20 Coffey RJ, Cahill D, Steers W, et al. Intrathecal baclofen for intractable spasticity of spinal origin: results of a long-term multicentre study. J Neurosurg 1993;78:226-32.

21 Becker WJ, Harris CJ, Long ML, et al. Long term intrathecal baclofen therapy in patients with intractable spasticity. Can J Neurol Sci 1995;22:208 -17.

22 Akman MN, Loubser PG, Donovan WH, et al. Intrathecal baclofen: does tolerance occur? Paraplegia; 1993;31:516-20. 